\title{
Design of a compact, reconfigurable, prosthetic wrist
}

\author{
Arthur Zinck $^{\mathrm{a}}$, Øyvind Stavdahl ${ }^{\mathrm{b}}$, Edmund Biden $^{\mathrm{a}}$ and Peter J. Kyberd ${ }^{\mathrm{a}, *}$ \\ ${ }^{a}$ Institute of Biomedical Engineering, University of New Brunswick, Fredericton NB, Canada \\ ${ }^{\mathrm{b}}$ Department of Engineering Cybernetics, Norwegian University of Science and Technology - NTNU, \\ Trondheim, Norway
}

\begin{abstract}
The design of a prosthetic wrist is the result of compromises between the function and the practicality of the device. Conventional prosthetic wrists use a single degree of freedom to produce pro/supination of the hand. It has not been demonstrated that this is the most functional alignment for a single axis. Previous work by the authors suggests that if the wrist must have only one rotatory axis then a more oblique orientation would be more functional. To test this idea, a compact wrist with a single axis and spherical design has been made that will allow any axis of rotation to be selected and the functional performance of the resulting arm be tested.
\end{abstract}

Keywords: Prosthetic wrist, brushless commutation

\section{Introduction}

The role of the wrist in prehension is to place the hand in the appropriate orientation to allow it to perform the grasping and manipulation [13,16]. To lose the use of the wrist is to impair the person significantly. In the majority of prosthetic fitting and research, the wrist has been overlooked. The primary reasons for this is that any prosthesis needs be practical. This means that the wrist has to be light and compact enough to be carried at the distal end of the arm, irrespective of the level of loss. It must also be easy to control to allow wearers to use the device simply, without needing to concentrate on the device and how it is controlled. Any moving prosthetic wrist adds to the mass and cost of the finished limb, and there is a perceived lack of functional improvement when it is supplied [13]. If the hand cannot be positioned simply then it is likely that the operator will not use it.

\footnotetext{
*Corresponding author. E-mail: pkyberd@unb.ca.
}

Conventional control of electric prostheses is through the use of myoelectric signals from the muscles of the forearm of the operator. Electromyography is the amplification of the electrical signals associated with muscular contraction, and it is used to control each motion within the prosthesis. This control can be very limited due to the lack of useable muscle sites. If this is the case then the device is controlled slowly and in a sequential manner; the user rotates the wrist, then switches and opens the hand [18]. If there is a need to adjust the wrist's orientation the user must switch back to the wrist before returning to the hand to close it. Hence the operation can be cumbersome, and using some other part of the body to position the hand, for example raising the arm, might be perceived as easier and quicker. Therefore, adding additional degrees of freedom does not necessarily add function. Pattern recognition of many muscle signals is a possible solution for some persons, (once the technology and techniques have been refined), [2, 7, 15]. This only works if there are enough muscle sites to give a pattern. 
Every person's loss is unique and for some users have a very limited number of muscle sites, so even when the technology improves it is likely that simpler control solutions will continue to be necessary. This paper looks at a design that investigates this problem.

\subsection{Use and replacement of the human wrist}

The natural wrist and forearm have a combined three degrees of freedom which can be utilized in tandem, giving the full range of motion of the joint. The wrist has two degrees of freedom: flexion/extension and ulnar/radial deviation. The third degree of freedom is pro/supination, which is properly a function of the forearm and not the wrist, but since this flexibility is lost when the person suffers an amputation anywhere along the forearm it is regarded as part of the functionality that is relevant for a prosthetic wrist to replace, Fig. 1.

In order to create prosthetic designs that are appropriate for the requirements of the user population, there needs to be an understanding of how a human performs a task and so how it is changed through use of aids such as prosthetic limbs. One consequence of the limits imposed when a prosthesis is used, is the need for compensatory motions by other parts of the body. These compensatory movements cause abnormal stress which may lead to repetitive strain injuries (RSI) [8].

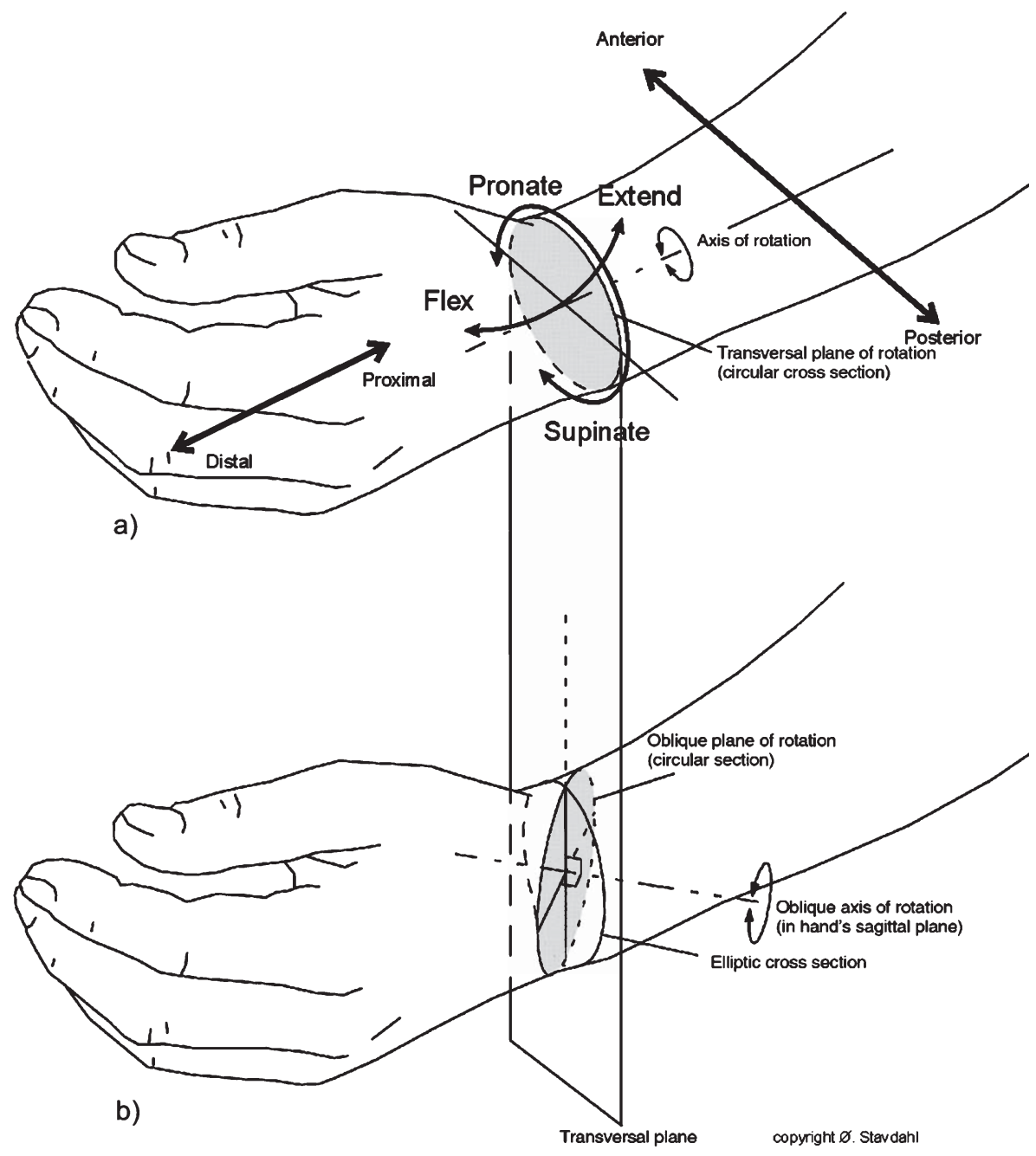

Fig. 1. a) The axes for the human wrist, definitions of terms used, including the usual axis for prosthetic wrists. b) Axis selected for the oblique wrist mechanism. 


\subsubsection{The physical effect of using a prosthesis}

The choice to use a prosthesis (or not) is a personal one, and based on many factors. When people perform a physical task, they tend to attempt to minimise the physical and mental effort in making the motion, by limiting ranges of motion, forces and the number of times they are performed. Compensatory movements are the extra movements that are required to perform the same task when some of the actions are not possible, due to injury or absence, such as the motions that may be required when a person uses a prosthesis because they have little or no wrist function. These extra movements may include increased elbow flexion/extension, more shoulder elevation; and more humeral rotation. Although the movements allow the task to be completed simply, these additional actions can cause other problems, such as joint pain and injury due to the increased use of the joints and muscles; so called over-use or repetitive strain injuries. Over-use injuries occur when joints and muscles are used over wide ranges, to generate large forces or perform the task for long times without time to rest and recover. The results can be debilitating and reduce the use of the joint in question [8]. When a person is using a prosthesis, these compensation motions are not restricted to the arm with the prosthesis, but can occur at any of the other joints of the body [11, 12, 19]. For example; although the lack of the ability to flex the wrist may cause users to raise their elbow to position the hand, they may also achieve greater range through bringing the object across the mid-line with the other hand, or lean toward the object to raise the elbow that way. Each and every one of these joints is then under threat from an over-use injury. If the person's motions are already limited by their absence of a hand, the additional discomfort of RSI in some other joint can only make their plight worse. Thus it should be the aim of any prosthesis designer to reduce the compensations required when a new prosthesis is designed. This work addresses one aspect of this problem.

\subsection{The prosthetic wrist}

Commercial prosthetic wrists generally are limited to a single degree of freedom, and pro/supination is the most common axis of motion (probably due to it being easier to manufacture a reliable wrist with this motion). Many fitted wrists are passive, with a pro/supination action, while [1] estimated that 30-40\% of myoelectric fittings in the U.S. and Canada possess electric wrists.
This is in contrast with the experience at the limb centre of the Institute of Biomedical Engineering (IBME) at UNB, where this work was conducted. Although they specialise in the use of myoelectric hands and arms and potentially have a wider experience than many other centres in North America, currently there are no powered wrist prostheses fitted.

Commercially, the most common powered pro/supination wrist is from Otto Bock ${ }^{1}$. The wrist unit is round in cross section allowing for continuous rotation, which is unnatural, but some users do find it functionally useful. It also has a connector that allows the terminal devices to be easily changed [6].

After many years of inattention, prosthetic wrists designs are currently enjoying greater interest with many more single degree of freedom (DOF) and two degree of freedom designs being designed. Examples include two degrees of freedom from $[4,5,10,14]$.

\subsection{Motivation}

Given the circumstance that designs of a practical wrist rarely have more than one axis, [16] explored which single axis might represent the ideal 1-DoF approximation of the healthy wrist's functional workspace. With a unilateral absence, the dominant arm is invariably the sound limb, which relegates the prosthesis to a support role [3]. Therefore Stavdahl's research considered the single degree of freedom wrist in tasks where the prosthesis was in the non-dominant role only. While pro/supination is the usual choice for powered wrists, no rational for this arrangement is ever stated, so it was proposed that a rotator set on some oblique plane may increase functionality [1].

In order to validate the concept, Stavdahl assessed eight right handed healthy subjects (21 to 64 years) during the performance of 15 different Activities of Daily Living (ADLs). The ADLs were selected on the basis of a pilot study which explored the compensatory movements of the elbow, shoulder and torso with in persons who have a trans-radial limb loss [16]. From this the optimal single axis of rotation across ADLs was found to go through the wrist from a dorsal-lateral position proximally to a ventral-medial (palmar) position distally, forming an angle of approximately $11^{\circ}$ with the forearm and $7^{\circ}$ with the sagittal plane. Further-

\footnotetext{
${ }^{1}$ Otto Bock HealthCare Deutschland GmbH and Co. KG, MaxNäder-Straße 15, D-37115 Duderstadt, Germany, Phone +49-55 27 848-0
} 
Table 1

Design Criteria used for the oblique wrist

\begin{tabular}{|c|c|c|c|}
\hline Criteria & Value & Dimension & Note \\
\hline Length & $<65$ & $\mathrm{~mm}$ & \\
\hline Mass & $<100$ & g & \\
\hline Speed & $\geq 1.42$ & $\operatorname{rad~s}^{-1}$ & $13.5 \mathrm{rpm}$ \\
\hline Stall Torque & $\geq 35$ & $\mathrm{mNm}$ & \\
\hline Adjustment & Oblique plane & & Adjust by clinicians, not by user \\
\hline $\begin{array}{l}\text { Diameter in the } \\
\text { transverse plane }\end{array}$ & 40 & $\mathrm{~mm}$ & $\begin{array}{l}\text { Less than or equal to the diameter } \\
\text { of the narrowest part of a healthy } \\
\text { adult male wrist }\end{array}$ \\
\hline
\end{tabular}

more the axis was displaced $19^{\circ}$ with respect to the 3rd metacarpal (defined as the proximal-distal axis of the hand) and $13^{\circ}$ with respect to the palmar plane of the hand. Figure 1 shows a somewhat neutral wrist posture corresponding to this kinematic model; note the presence of a slight static wrist extension, i.e. a rotational offset between the hand and the forearm perpendicularly to the wrist's axis of rotation. Only small differences were found in the optimal kinematic parameters for various groups of subjects based on age and sex, but significant variations were found between the different ADLs. These variations call for further investigations, and point to the need for an experimental prosthetic wrist which can be adjusted to take on different kinematic properties. The present study demonstrates the viability of such a design, and provides a test bed for further investigations of optimal wrist prosthesis kinematics.

\subsection{Design Criteria}

To be practical, any prosthetic joint needs to be as compact as possible. It should take up as little space as possible and require as little power to operate and the least amount of effort to use, be it mental or physical. As a starting point for the design, the criteria were based on the only successful mechanisms available, the existing commercial products, Table 1 . As the prosthesis is to be used in a support role its torque need not be large, it will merely position the hand with the correct orientation for the hand to be used to assist the natural hand. The wrist needs to be reconfigureable so that it could be used to test any alignment, so it must be based around a sphere with adjustment at either end. It must also fit within a conventional prosthetic system and take the standard inputs so that to the user the only difference is the wrist itself.

\section{Results}

The new wrist was designed as a single degree of freedom rotation device with a user controllable motorised axis that could be pre-positioned at different orientations by the prosthetist adjusting the alignment both at the proximal and at the distal ends of the wrist, [17]. This design allows the wrist to be versatile in testing different oblique plane orientations as well as standard pro/supination rotator. This is useful for allowing clinical measurement of the function to be made. These features remove variables such as speed, torque, size and weight differences between different wrists. When comparisons are performed this wrist can behave as a standard wrist or an oblique angle wrist while leaving all other parts of the prosthetic fitting unchanged.

The wrist was conceived to be part of a complete arm system so that all other structures (batteries, amplifier/electrodes for control with additional prosthetic hand positioned via a socket on the residuum), are as conventional as possible. Input and output devices are housed externally to the wrist and communicate via a computer bus [9], or conventional analogue signalling.

The entire wrist fits within a sphere with overall diameter $45.5 \mathrm{~mm}$, with adjustment slots at both ends and $t$-shaped nuts to allow the change of the orientation of plane of rotation. The end caps of the mechanism are domed to facilitate the adjustment, with a central housing made from a proximal and a distal section of $40 \mathrm{~mm}$ diameter, that hold the drive train and electronics. The communications bus runs through the centre of the wrist via slip rings. The wrist rotates on a centre bearing, giving the wrist its single degree of freedom, (general assembly can be seen in Fig. 2).

Adjustment of the orientation of the wrist is only to be undertaken at the clinic, the idea being to allow the angle to be set and then the user allowed to operate the 

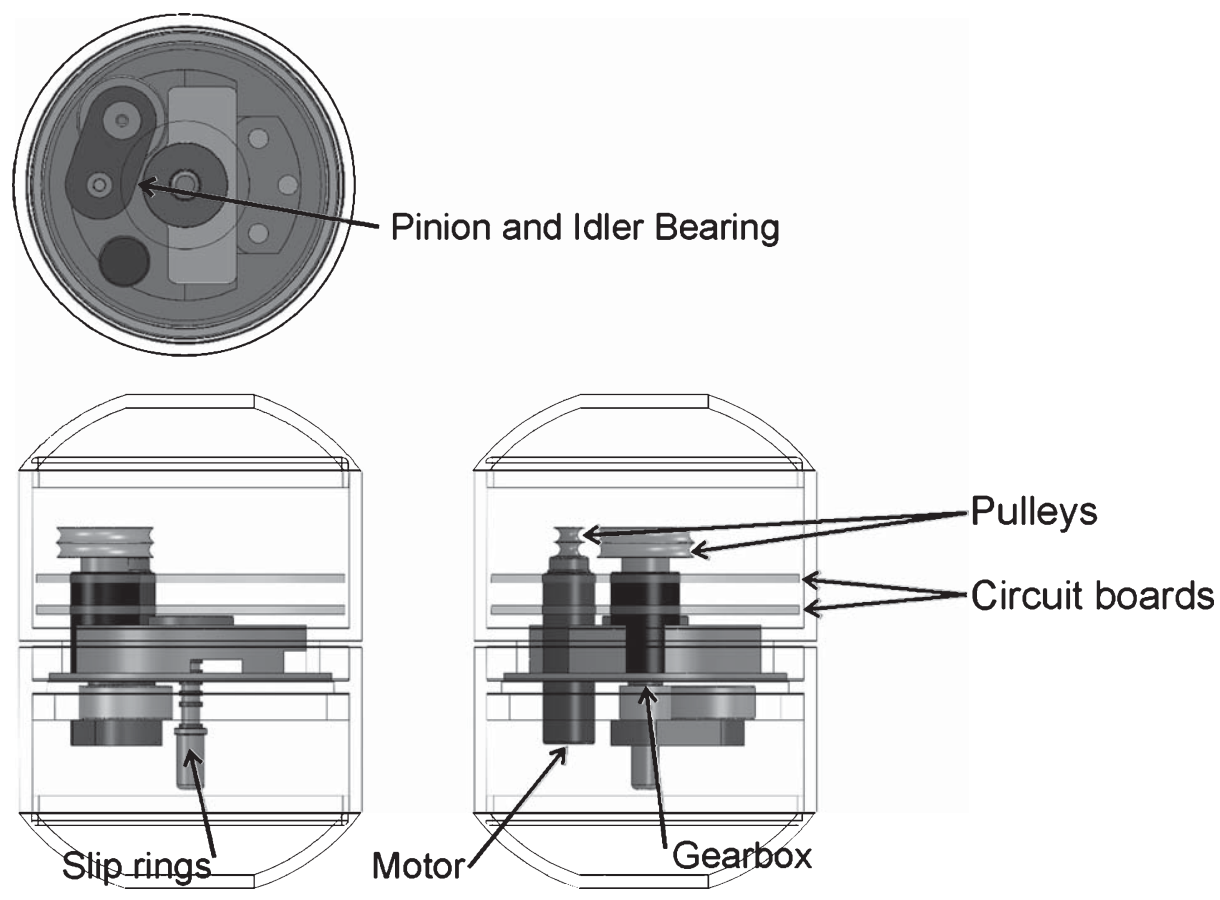

Fig. 2. The overall design of the reconfigruable wrist. End cap domes are displaced proximally and distally for clarity.

wrist at this angle. The orientation is set by loosening the t-nuts and moving the wrist along the slot before tightening them again. Once tightened the wrist is fixed in this alignment.

The main body of the wrist houses the drive motor, drive train and the controller. The electronic controller was designed to fit on the four surfaces of the two $36 \mathrm{~mm}$ diameter circuit boards that stack one on top of the other, Fig. 3, and fit into the proximal wrist section. The motor and the transmission fit through the both circuit boards reducing the overall space requirements, Fig. 4.

The distal section houses a 120-tooth internal ring gear that was press fit inside the distal section of the housing. This section also contains slip rings, with a brush block attached to the proximal section to allow power and data transmission through the wrist to the hand.

\subsection{Drive train}

The wrist is driven with a brushless DC motor. The required reduction ratio was accomplished using three separate stages consisting of a belt primary stage, a planetary gear second stage and a ring and pinion

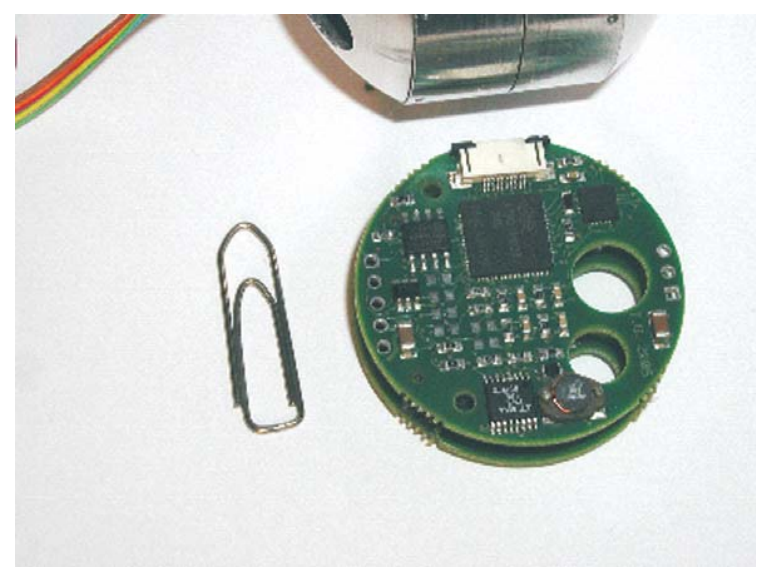

Fig. 3. The two $36 \mathrm{~mm}$ diameter circuit board designed to control the wrist. They stack on top of each other, the holes are to allow the motor and gearbox to pass through.

third stage, Fig. 5. Details of the drive train are in Table 2.

\subsubsection{Reduction}

Metal gears were discounted for the first stage as it is the fastest part of the drive chain and likely to be the most noisy. Thus a potentially quieter, rubber belt was 


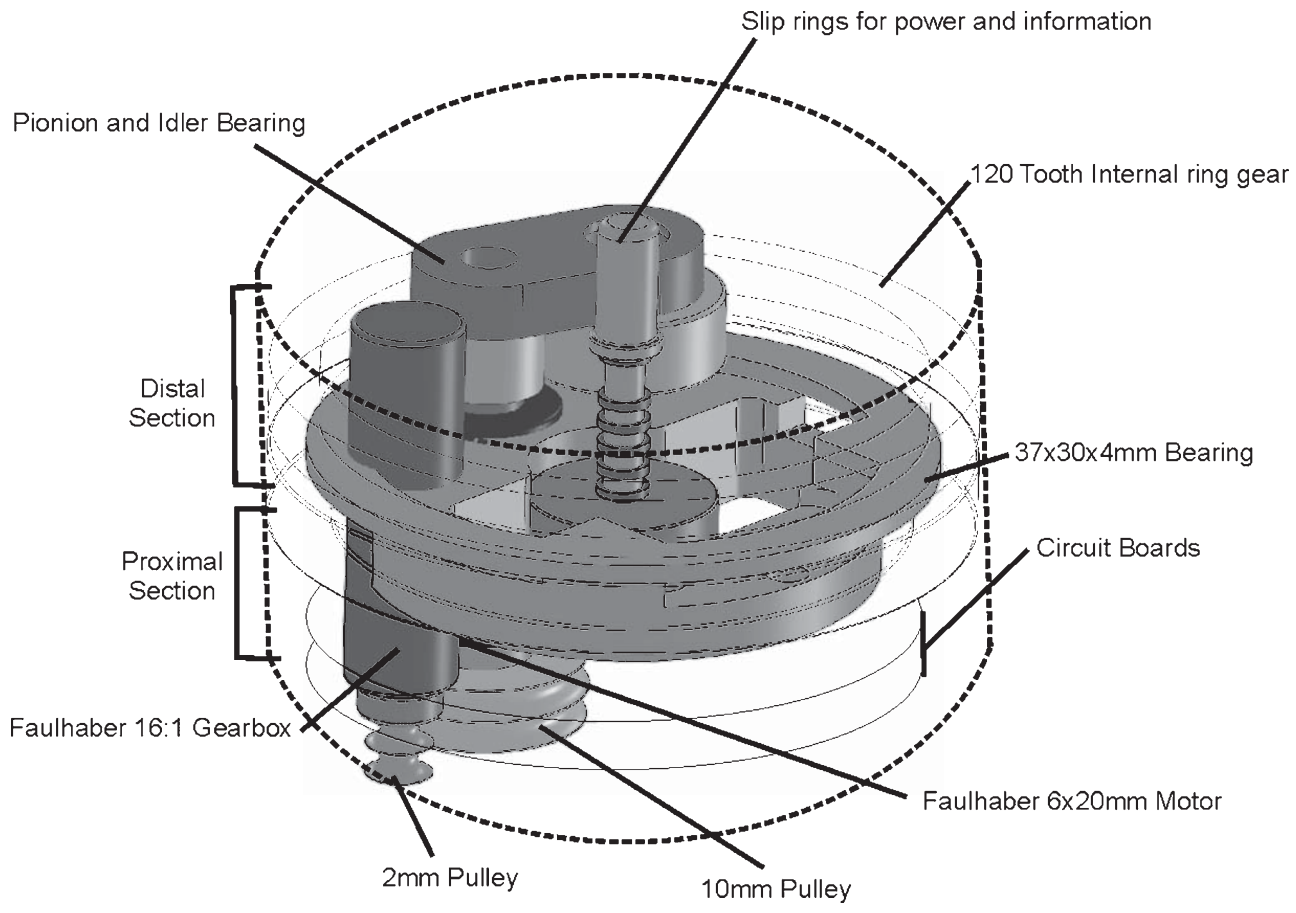

Fig. 4. Placement of the internal components of the wrist.

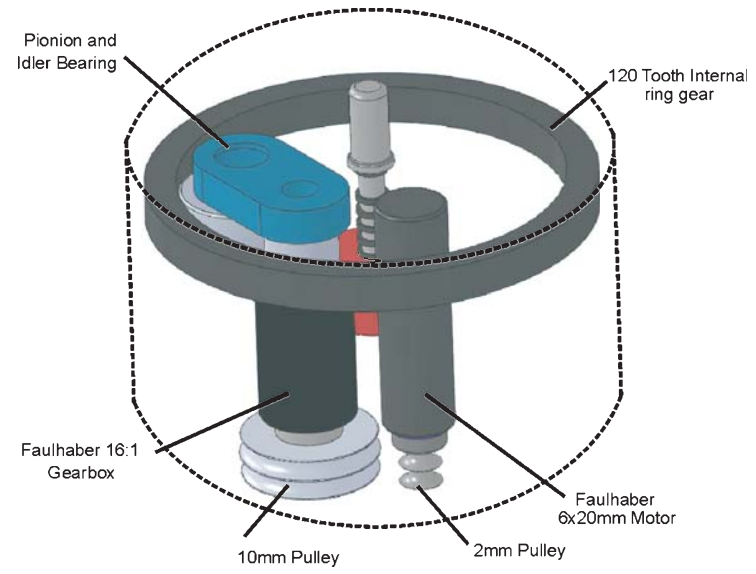

Fig. 5. Transmission assembly showing the motors the ring and pinion and the pulleys for the first reduction stage.

chosen. Noise is an important concern to prosthesis users who do not wish to attract attention.

The final gear stage was made up of pinion, a small idler gear, and an internal ring gear. The idler gear allowed the motor and planetary gearbox to be located optimally within the space of the wrist.
The third stage increased the overall reduction ratio to $800: 1$ which increases the torque to $584.0 \mathrm{mNm}$ (before efficiency losses are considered) and reduces the speed down to $58.75 \mathrm{rpm}$ (also before losses). Stall torque measured with a dynamometer was closer to $60 \mathrm{mNm}$.

\subsubsection{Controller}

The control of the wrist is achieved by a microprocessor controller that is mounted in the proximal part of the wrist. Brushless commutation was achieved using the ATiny2313 microcontroller from Atmel. Position/velocity control is achieved using internal feedback from the brushless motor unit and the angle of the wrist is measured using a permanent magnet attached to the slip ring unit and a PCB-mounted Hall effect device. A simple PID controller suffices for this application. For testing, the user can either instruct the wrist or the hand, depending on which is selected. The selection can be performed using conventional EMG switching with a co-contraction of the two muscle groups. Other control strategies can easily be implemented through re-programming of the controller. 
Table 2

Details of the drive train

\begin{tabular}{lrcccl}
\hline $\begin{array}{l}\text { Stage } \\
\text { ratio }\end{array}$ & $\begin{array}{c}\text { Stage } \\
\text { gear }\end{array}$ & $\begin{array}{c}\text { Cumulative } \\
\text { ratio }\end{array}$ & $\begin{array}{c}\text { Velocity after } \\
\text { each stage } \\
(\mathrm{rpm})\end{array}$ & $\begin{array}{c}\text { Torque after } \\
\text { each stage } \\
(\mathrm{mNm})\end{array}$ & Notes \\
\hline Motor & $1: 1$ & $1: 1$ & 47000 & 0.73 & Faulhaber 0620 K 006 B \\
First & $5: 1$ & $5: 1$ & 9400 & 3.65 & Belt \\
Second & $16: 1$ & $80: 1$ & 587.5 & 58.4 & Faulhaber 08/1 K - Planetary Gear \\
Third & $10: 1$ & $800: 1$ & 58.8 & 584.0 & $\begin{array}{l}\text { Ring and pinion } \\
\text { 303 stainless steel } \\
\end{array}$ \\
& & & & Pitch: 96 \\
& & & & Face width: $3.175 \mathrm{~mm}$ \\
Pressure angle: 200
\end{tabular}

Table 3

Results of the design, compared with target values, all targets were met or exceeded. (Stall torques were measured using a cable attached to a dynamometer, result of eleven trails are shown

\begin{tabular}{|c|c|c|c|}
\hline Criteria & Target & Value & Dimension \\
\hline Length & $<65$ & 65 & $\mathrm{~mm}$ \\
\hline Mass & $<100$ & 87 & $\mathrm{~g}$ \\
\hline Speed & $\geq 1.42$ & $4.3 \pm 0.1$ & $\operatorname{rad~s}{ }^{-1}$ \\
\hline Stall torque & $\geq 35$ & $59.6 \pm 3.7$ & $\mathrm{mNm}$ \\
\hline $\begin{array}{l}\text { Diameter in the } \\
\text { transverse plane }\end{array}$ & 40 & 40 & $\mathrm{~mm}$ \\
\hline
\end{tabular}

\subsection{Specifics}

The resulting design aimed at allowing this concept to be tested with prosthesis users, and it met the above criteria, see Table 3. While the losses were large the device was still able to produce the required torques.

The wrist is based on a $45.5 \mathrm{~mm}$ hemisphere (Fig. 6a) allowing adjustment with very little offset of the hand with respect to the forearm, even when the plane is set at extreme angles. The wrist mechanism measures $45.5 \mathrm{~mm}$ long with a diameter of $40 \mathrm{~mm}$ (without the ends to interface to the rest of the prosthesis) and $65 \mathrm{~mm}$ long when complete for clinical application (Fig. 6b).

\section{Discussion}

In order to test ideas about prosthetic control or application it is necessary to construct devices that are compact enough to be practical. This device has the capability of testing ideas of the orientation of a single axis or rotation at the wrist without changing the properties of any other part of the device, ensuring that all other factors remain the same, hence making test results easier to interpret.

The next stage of development is for this wrist to be used by persons performing a series of bimanual activities of daily living which were refined by the authors and have been tested on able bodied subjects and those
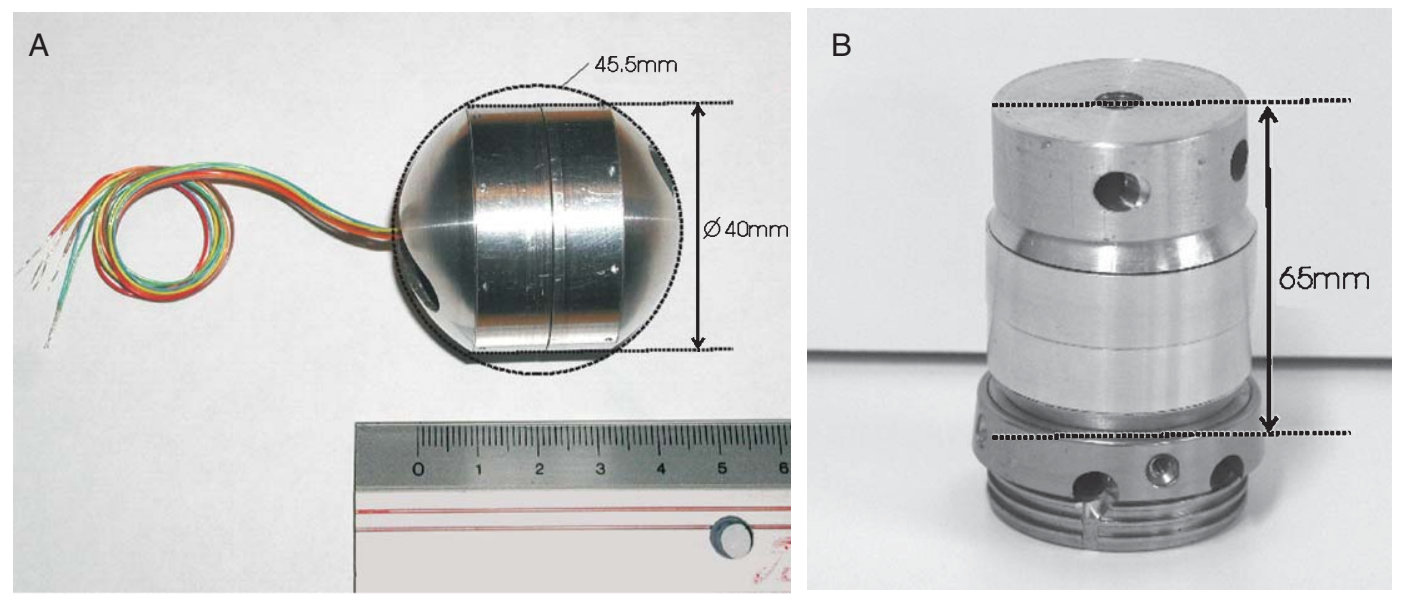

Fig. 6. Complete wrist system (a) Completed wrist showing overall diameter of $45 \mathrm{~mm}$ and cross section $40 \mathrm{~mm}$. (b) Complete wrist prosthesis showing the mounting to the user (bottom lamination ring) and the upper end to the prosthetic hand. Overall length $65 \mathrm{~mm}$. 
with conventional prostheses to allow direct comparisons to be made between the compensatory actions of a person with a conventional pro/supination wrist and one at an oblique angle. The experiments will be to test the subjects performing the bimanual activities of daily living with the wrist in the standard pro/supination orientation and with it set to the oblique angle and to observe and record the levels of compensation required at the effected arm and in the rest of the body.

\section{Acknowledgements}

The authors would like to thank various organisations that have supported this work: The Canadian Foundation for Innovation; Canada Institutes of Health Research; New Brunswick Innovation Foundation. Also Adam Clawson for assistance in the preparation of the drawings.

\section{References}

[1] R.D. Alleym and H.H. Sears, Powered upper limb prosthetics in adults, powered upper limb prostheses control, Implementation and Clinical Application, Springer-Verlag, New York, 2004, pp. 117-145.

[2] C. Almström, An electronic control system for a prosthetic hand with six degrees of freedom, Technical Report 1:77, Research Laboratory of Medical Electronics, Chalmers University of Technology, 1977.

[3] D.J. Atkins, D.C.Y. Heard and W.H. Donovan, Epidemic overview of individuals with upper-limb loss and their reported research priorities, Journal of Prosthetics and Orthotics 8(1) (1996), 2-11.

[4] K.J. De Laurentis and S. Phillips, Design of a powered twoDoF prosthetic wrist, Proceedings of 12th World Congress, page 524, International Society of Prosthetics and Orthotics, 2007.

[5] H. Dietl, Prosthesis Control Based on TMR - Proceesings of MEC'08 - Measuring Success in Upper Limb Prosthetics, Symposium, University of New Brunswick, Fredericton, Canada, 2008, pp. 166-170.

[6] B. Engstrom, and C. Van de Ven, Therapy for Amputees, Third Edition, Chruchill Livingstone, ISBN 04405975 6, 1999.

[7] P. Herberts, C. Almström, R. Kadefors and P. Lawrence, Hand prosthesis control via myoelectric patterns, Acta Orthopaedica Scandinavia 44 (1973), 389-409.
[8] P.S. Kidd, C. McCoy, L. Steenbergen, Repetitive Strain Injuries in Youths, Journal of the American Academy of Nurse Practitioners 12(10) (2000), 413-426.

[9] P.J. Kyberd, A.S. Poulton, L. Sandsjö, S. Jönsson, B. Jones and D. Gow, The ToMPAW modular prosthesis - A platform for research in Upper Limb prosthetics, Journal of Prosthetics and Orthotics 19(1) (2007), 12-21.

[10] P. Kyberd, L. Goudreau, E. Lemaire, A Two Degree of Freedom Powered Prosthetic Wrist, Proceedings of 12th World Congress, International Society of Prosthetics and Orthotics, 2007 , pp. 523

[11] J.S. Landry, Optimal Fixed Wrist Alignment for BelowElbow, Powered Prosthetic Hands, Masters, Department of Mechanical Engineering, University of New Brunswick, 2000 .

[12] B. MacPhee, Examining the Prosthetic Function and Body Behaviour of Prosthesis Users Performing Activities of Daily Living, Masters, Department of Mechanical Engineering, University of New Brunswick, 2007.

[13] J.W. Michael, Externally Powered Prostheses for the Adult Transradial and Wrist Disarticulation Amputee. In: H. Meier Robert and J. Atkins Diane (eds.), Functional Restoration of Adults and Children with Upper Extremity Amputation Demo Medical Publishing Inc, New York, 2004, pp. 187-197.

[14] D.H. Plettenburg, The Wilmer 2-DOF Wrist Prosthesis for toddlers, Proceedings of 12th World Congress, International Society of Prosthetics and Orthotics, 2007, pp. 522.

[15] E. Scheme and K. Englehart, A flexible user interface for rapid prototyping of advanced real-time myoelectric control schemes, Proceedings of MEC'08 - Measuring Success in Upper Limb Prosthetics, pages 150-155. UNB, IBME, Fredericton, New Brunswick, Canada, 2008.

[16] Ø. Stavdahl, Optimal wrist prosthesis kinematics: Three dimensional rotation statistics and parameter estimation, Ph.d. thesis, Norwegian University of Science and Technology, Trondheim, Norway, 2002

[17] Ø. Stavdahl, A. Zinck, P. Kyberd, W. Hill and G. Bush, A prosthetic wrist with an oblique plane of motion to optimise device weight, size and functionality. p.521 Proceedings of 12 th World Congress, International Society of Prosthetics and Orthotics, 2007, pp. 521

[18] T.W. Williams, Control of powered upper extremity prostheses. In: H. Meier Robert and J. Atkins Diane (eds.), Functiona Restoration of Adults and Children with Upper Extremity Amputation, Demo Medical Publishing Inc, New York, 2004 pp. 207-224.

[19] A.L. Zinck, Investigation of Compensatory Movements in Prosthesis Users and the Design of a Novel Wrist, Masters Department of Mechanical Engineering, University of New Brunswick, 2008. 

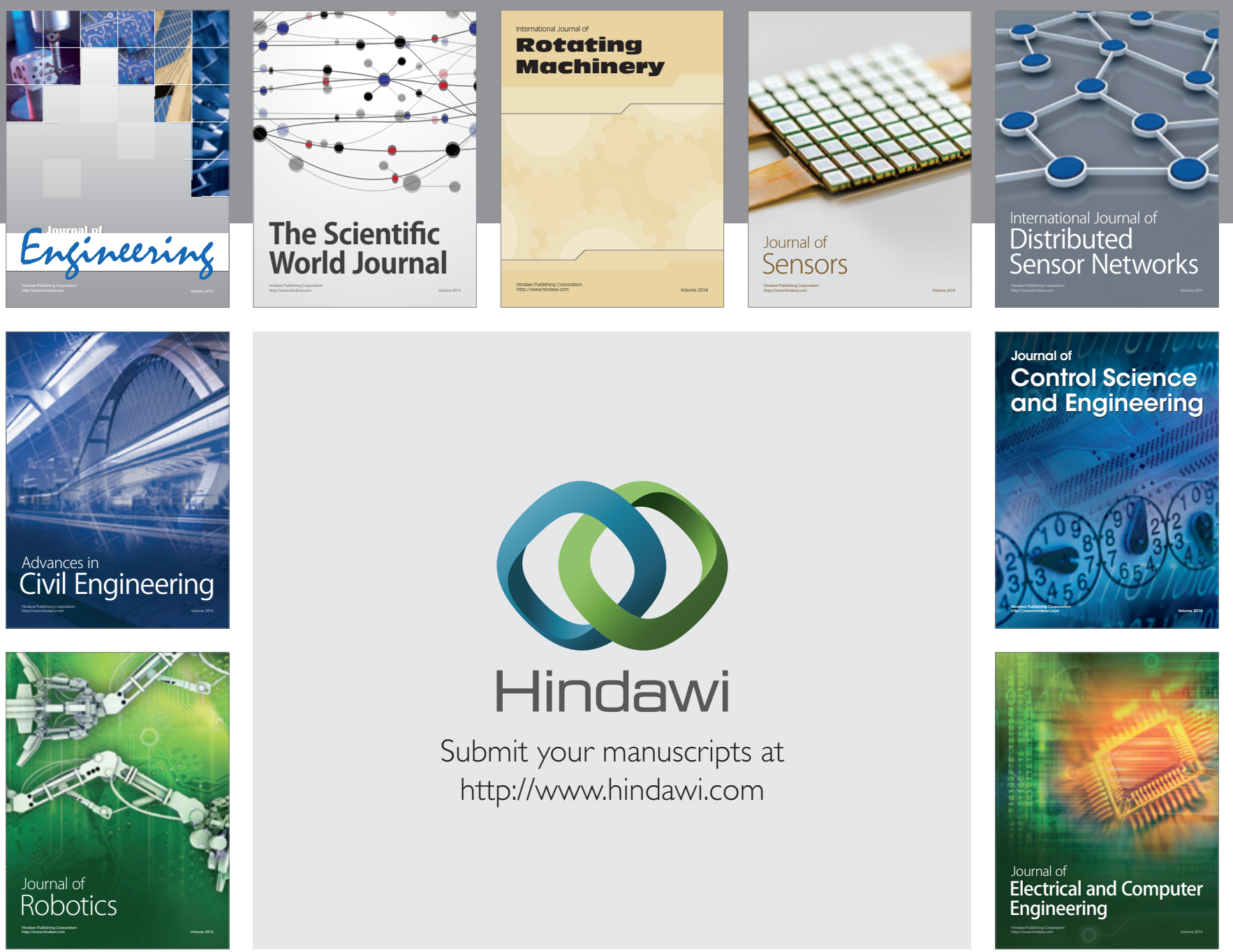

Submit your manuscripts at

http://www.hindawi.com
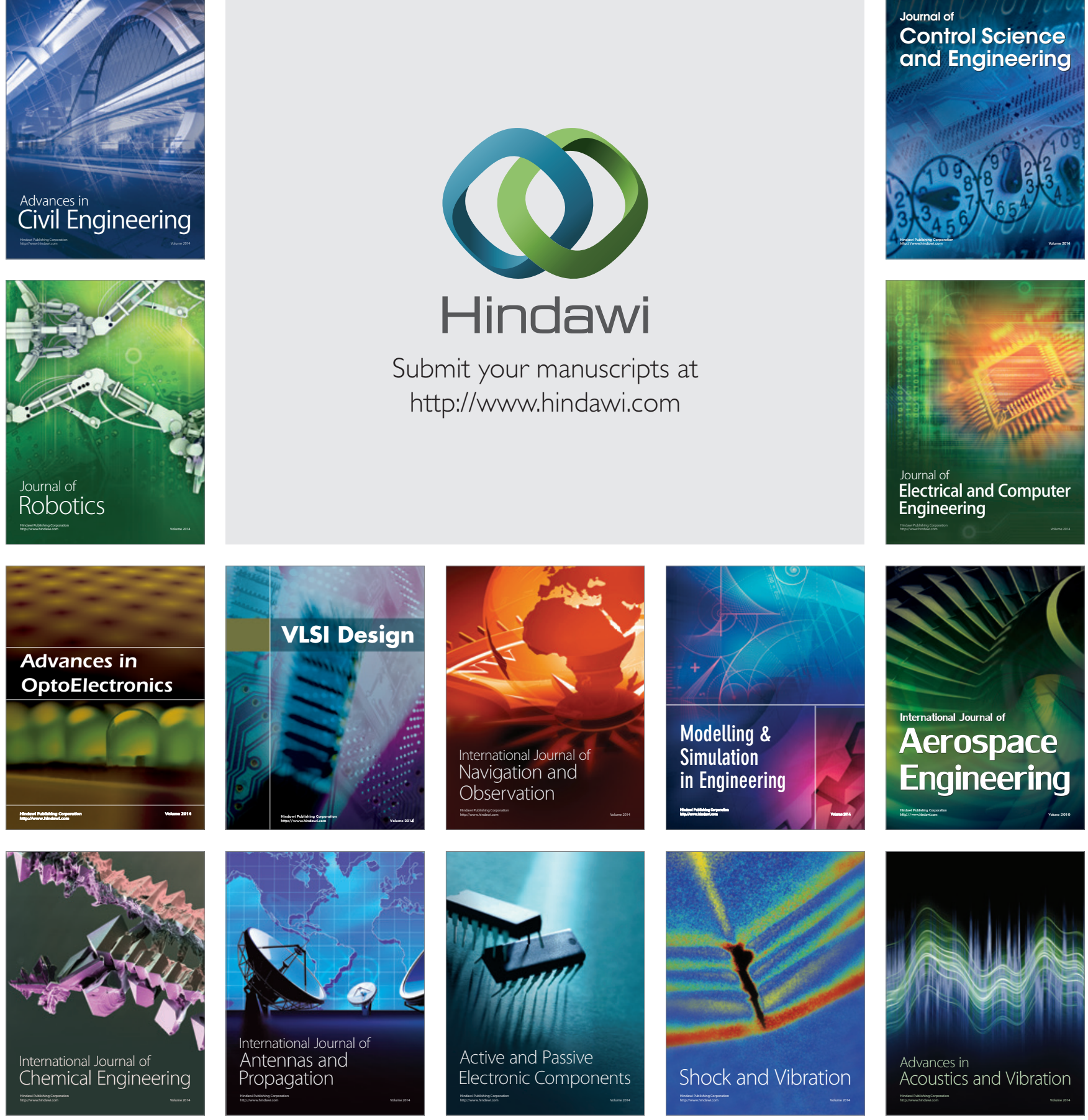